\title{
Estudio de la composición y estructura del bosque andino localizado en Potrero Grande, Chipaque (Colombia)
}

\section{Study of the composition and structure of the andean forest in Potrero Grande, Chipaque (Colombia)}

\author{
Lorena Cortés-Ballén ${ }^{1}$; Shirley Camacho-Ballesteros ${ }^{2}$; Mauricio Matoma-Cardona ${ }^{3}$
}

1Ecóloga, Esp. M.Sc. Jardín Botánico de Bogotá José Celestino Mutis. Bogotá, Colombia; e-mail: lacortes@jbb.gov.co; (D) https://orcid.org/0000-0002-32974905

${ }^{2}$ Administradora Ambiental, Esp. Jardín Botánico de Bogotá José Celestino Mutis. Bogotá, Colombia; e-mail: scamacho@jbb.gov.co; (D) https://orcid. org/0000-0002-0219-0560

${ }^{3}$ Ing. Forestal. Jardín Botánico de Bogotá José Celestino Mutis. Bogotá, Colombia; e-mail: mauriciomatomacardona@gmail.com; (D) https://orcid.org/00000001-5203-6847

Cómo citar: Cortés-Ballén, L.; Camacho-Ballesteros, S.; Matoma-Cardona, M. 2020. Estudio de la composición y estructura del bosque andino localizado en Potrero Grande, Chipaque (Colombia). Rev. U.D.C.A Act. \& Div. Cient. 23(1):e1483. http://doi.org/10.31910/rudca. v23.n1.2020.1483

Artículo de acceso abierto publicado por Revista U.D.C.A Actualidad \& Divulgación Científica, bajo una licencia Creative Commons CC BY-NC 4.0

Publicación oficial de la Universidad de Ciencias Aplicadas y Ambientales U.D.C.A, Institución de Educación Superior Acreditada de Alta Calidad por el Ministerio de Educación Nacional.

Recibido: Julio 22 de 2019

Aceptado: Febrero 28 de 2020

Editado por: Ingeborg Zenner de Polanía

\section{RESUMEN}

Los bosques de los Andes son grandes reservorios de biodiversidad y de endemismos y constituyen una ecorregión terrestre prioritaria, a nivel mundial; no obstante, la pérdida y degradación de hábitats, suscitada por los conflictos de uso, ha generado consecuencias irreparables para la calidad en el suministro de recursos y servicios de estos ecosistemas, siendo una de las principales amenazas para su conservación. Para contrarrestar esta situación, una eventual alternativa radica en estudiar la sucesión ecológica de bosques secundarios, con el fin de conocer la ecología y el ensamblaje de las comunidades vegetales y, con ello, favorecer la toma de decisiones en materia de restauración de estos ecosistemas. En este estudio, se analizó la composición y la estructura de la vegetación secundaria del Bosque Andino, ubicado en el municipio de Chipaque (Cundinamarca). Se establecieron dos parcelas de 0,1ha y una de 0,05 ha, abordando fragmentos de vegetación homogéneos; se hallaron 523 individuos, distribuidos en 69 especies, 36 géneros y 25 familias; los índices de diversidad evidenciaron tres comunidades vegetales diferentes, con baja dominancia y alta diversidad. Las densidades de individuos para clases diamétricas y alturas presentaron una tendencia a la distribución asimétrica positiva, evidenciando una regeneración activa; el Índice de Valor de Importancia señaló a las especies Clusia multiflora y Centronia brachycera, con mayor representatividad ecológica. La heterogeneidad florística encontrada proporciona conocimiento científico estratégico, para explorar diferentes alternativas de propagación e introducción de especies nativas, que resulten propicias para integrar en procesos de restauración, en áreas degradas de la región.

Palabras clave: bosque secundario; bosque Andino; biodiversidad; sucesión de plantas. 


\section{ABSTRACT}

The forests of the Andes are large reservoirs of biodiversity and endemism and constitute a priority land ecoregion worldwide. However, the loss and degradation of habitats caused by conflicts of use has generated irreparable consequences for the quality of the supply of resources and services of these ecosystems, being one of the main threats to their conservation. To counteract this situation, an eventual alternative lies in studying the ecological succession of secondary forests in order to know the ecology and assembly of plant communities, and thus, favor decision-making regarding the restoration of these ecosystems. In this study, the composition and structure of the secondary vegetation of the Andean Forest located in the municipality of Chipaque was analyzed. Two plots of 0.1 ha and one of 0.05 ha were established in homogeneous vegetation fragments; physiognomic variables were recorded for seedling, saplings and poles. There were 523 individuals distributed in 48 species, 36 genera and 25 families; the diversity indices showed three different plant communities with low dominance of species and high diversity of taxa. The densities of individuals for diameter classes and heights showed a tendency to positive asymmetric distribution, showing an active regeneration; the Value of Significance Index indicated the species Clusia multiflora and Centronia brachycera with greater ecological representativeness. The floristic heterogeneity found provides strategic scientific knowledge to explore different alternatives of propagation and introduction of native species in restoration processes in degraded areas of the region.

Keywords: secondary forest; andean forest; biodiversity; ecological succession.

\section{INTRODUCCIÓN}

Los Bosques Andinos son ecosistemas de montaña, con un amplio rango de elevación; se encuentran enmarcados entre los $2.400 \mathrm{~m}$ s.n.m. y 3.500m s.n.m. (Rangel et al. 1997), incluyendo los bosques de Robledal, boques altos y matorrales altoandinos. En la región Andina baja, este rango oscila entre los 2.400 a los $2.800 \mathrm{~m}$ s.n.m (Preciado, 2000) y en la región subandina, el rango varía de los 1.000 a $2.400 \mathrm{~m}$ s.n.m. (Rangel et al. 1997). Los reportes nacionales para la Evaluación de los Recursos Forestales Mundiales de la Organización de las Naciones Unidas para la Alimentación y la Agricultura FAO (2015) señalan que los Bosques Andinos representan, aproximadamente, el 24,9\% de los bosques en Colombia; el 31,98\%, en Ecuador; el 27,76\%, en Perú y el 20\%, en Bolivia.

Dadas sus características, estos ecosistemas son altamente sensibles al cambio, puesto que su establecimiento en relieves empinados genera erosión extrema, bajo un régimen de alta precipitación; sumado a esto, los fenómenos de aumento poblacional, deforestación, degradación, conversión de usos del suelo, principalmente, por la extensión de la frontera agropecuaria para ganadería vacuna, actividades mineras y cambio climático, generan la rápida reducción de su extensión superficial. (Bussmann, 2004; Sabogal et al. 2015; Armenteras et al. 2017).
Una buena parte de los bosques andinos, que hoy existen, se encuentran en mosaicos aislados, asociados a usos de suelo para agricultura y ganadería, con dinámicas de regeneración natural, que responden a procesos ecológicos estrechamente relacionados con la respuesta a cambios en variables ambientales y de intervención antrópica (Alvear et al. 2010).

El seguimiento a la sucesión de los bosques Andinos, de manera posterior a procesos de perturbación, es fundamental, para conocer la resiliencia de estos ecosistemas y su ecología. Este proceso responde a un reemplazo en la estructura, la composición taxonómica y los procesos funcionales que, a través del tiempo, generan un estado del ecosistema previo al disturbio, o bien, estados alternativos (Pickett \& White, 1985; Whitmore, 1998; Morin, 2011). El estudio de esta dinámica es una herramienta eficaz en restauración de comunidades de plantas, puesto que resulta determinante en el ensamblaje de la estructura y el funcionamiento de los ecosistemas (Guariguata \& Ostertag, 2001; Chazdon, 2003; Quesada et al. 2009; Morin, 2011).

El presente estudio, se enmarca en determinar la composición y la estructura de tres parcelas de vegetación secundaria, correspondiente al ecosistema de bosque Andino, ubicado en el municipio de Chipaque (Cundinamarca), caracterizado por procesos de disturbios, asociados al pastoreo y la agricultura.

\section{MATERIALES Y MÉTODOS}

Área de estudio. Se encuentra ubicada en la vereda Potrero Grande, del municipio de Chipaque, ubicado al suroriente del departamento de Cundinamarca, con alturas que oscilan desde los $2.470 \mathrm{~m}$ s.n.m., en el casco urbano, hasta 3.200m s.n.m., en el Boquerón de Chipaque.

Método. Se efectuaron tres levantamientos, respondiendo a: Parcela 1 (4²3'28.98'N, 74 4’33.69' O), Parcela 2 (4²3'32.74”N, $74^{\circ}$ 4'42.66”O) y Parcela 3 (4²3'53.59”N, 74 4’4.08”O) (Figura 1). La topografía del municipio, se caracteriza por el predominio de moderadas y fuertes pendientes, que van del $5 \%$, terreno plano; $30 \%$, terreno ondulado y el 65\%, terreno inclinado. En su geología, este municipio pertenece al Cretáceo, al grupo Villeta, de la conformación Chipaque (Guadalupe inferior); los mantos litológicos de la formación Chipaque son arcillas negras, esquirlas, calizas areniscas y areniscas de grano fino. Pertenece a la cuenca alta del río Meta y a la subcuenca del río Negro y cuenta con una extensión territorial de 13.945ha (Alcaldía Municipal de Chipaque Cundinamarca, 2000).

En el municipio, se desarrollan actividades de agricultura y de ganadería, integradas en un mosaico de cultivos, pastos, especies naturales y bosques densos altos. Las tres parcelas fueron establecidas en zonas de cobertura boscosa, ubicadas sobre la microcuenca fruticas, una de sus principales fuentes hídricas (Alcaldía Municipal de Chipaque Cundinamarca, 2000). La precipitación promedio multianual, entre 1981 y 2010 , es de $958,5 \mathrm{~mm}$ al año y la temperatura promedio multianual, entre 1981 y 2010 , es de $11,2^{\circ} \mathrm{C}$, de acuerdo con las estaciones metereológicas ubicadas en Bogotá, D.C. y en 


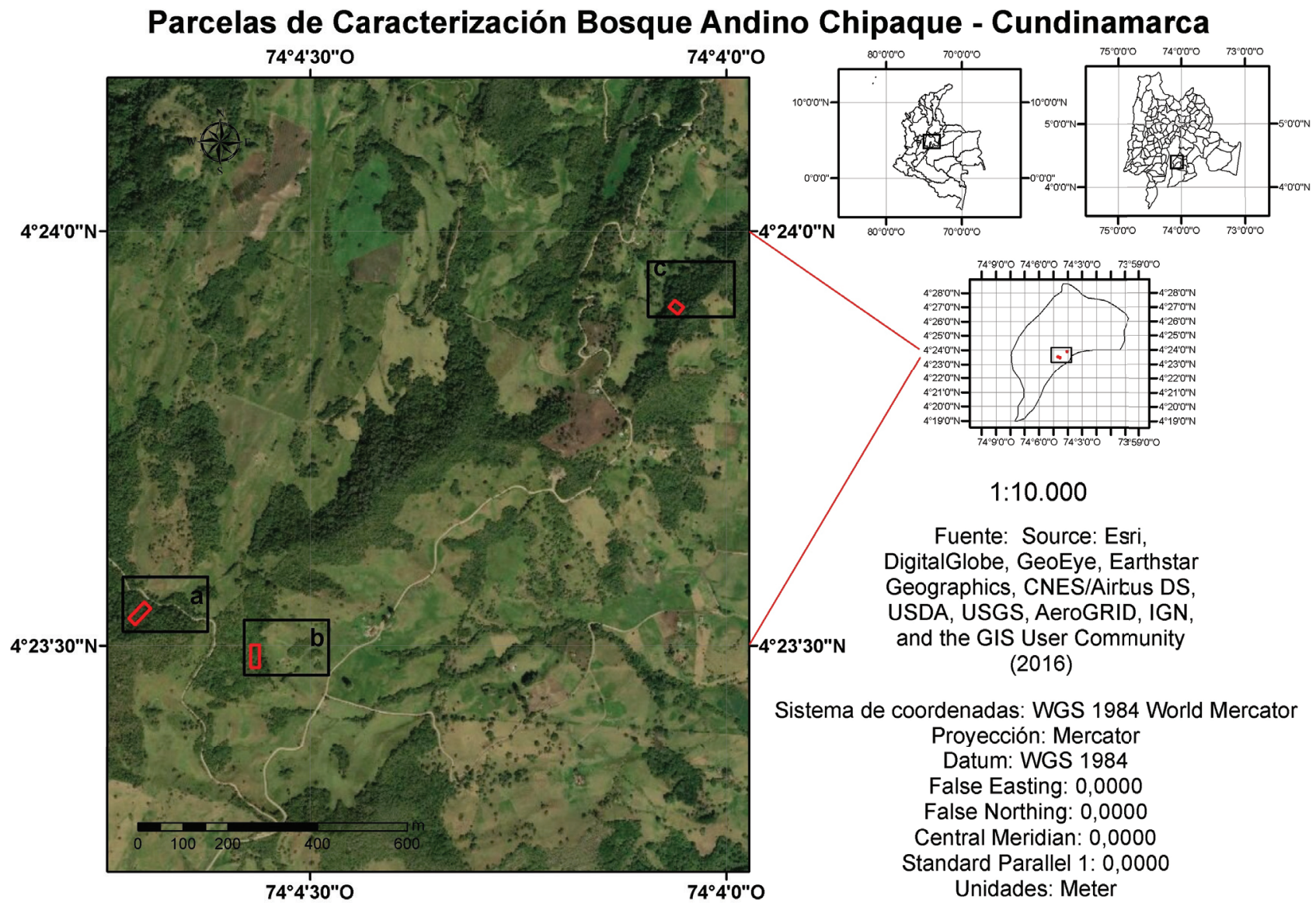

Figura 1. Localización geográfica del área de estudio, vereda Potrero Grande, Municipio de Chipaque, Cundinamarca (Colombia). a. Parcela 2; b. Parcela 1; c. Parcela 3.

los municipios de Chipaque, Caqueza, Choachi, Fosca, Gutiérrez, Ubaque y Une (IDEAM, 2018).

El método empleado respondió a un muestreo estratificado, que se llevó a cabo durante abril, mayo y junio de 2018. Se establecieron dos parcelas de 50 × $20 \mathrm{~m}(0,1$ ha $)$ y una parcela de $25 \times 20 \mathrm{~m}(0,05 \mathrm{ha})$, de acuerdo con las unidades mínimas de muestreo, propuesta por Rangel \& Velázquez (1997), para levantamientos de vegetación en bosques montanos.

Se realizó la medición de variables de altura total, diámetro a la altura del pecho y cobertura de copas, mediante la proyección de los diámetros de sus ejes, para fustales (DAP $\geq 10 \mathrm{~cm}$ ). Cada parcela, se subdividió en 5 cuadrantes de 5 x $5 \mathrm{~m}$, para la medición de individuos latizales $(2,5 \mathrm{~cm} \geq \mathrm{DAP}<10 \mathrm{~cm})$ y en 5 cuadrantes de $1 \times 1 \mathrm{~m}$, para la medición de brinzales y renuevos $(<2,5 \mathrm{~cm}$ y $\mathrm{HT}<0,3 \mathrm{~m})$, adaptando la metodología descrita por Sáenz \& Finegan (2000), para el muestreo de regeneración natural en bosques tropicales. A partir del levantamiento de información de las tres unidades de muestreo, se elaboró una matriz con los valores de altura y de DAP.
La identificación taxonómica fue realizada mediante la verificación de ejemplares, con colecciones botánicas virtuales y en medio físico, por medio del uso de claves taxonómicas, así como la consulta a expertos. Los ejemplares fueron depositados en el Herbario del Jardín Botánico de Bogotá José Celestino Mutis (JBB).

Análisis de información. Se evaluó la composición y la estructura, a nivel de familias y de especies, para cada transecto. Para estimar la diversidad de cada comunidad en particular, es decir, la diversidad Alpha (a) y Beta (ß), se evaluaron los índices de Shannon-Wiener, Simpson y Bray-Curtis (Moreno, 2001). Los datos fueron procesados mediante el programa Past 3.22 (Hammer et al. 2001).

A nivel estructural, se realizó un análisis de distribución de clases, según la altura y el DAP. Para este fin, se generaron intervalos o categorías de análisis de los estratos verticales en el bosque. Igualmente, se calculó el Índice de Valor de Importancia (IVI) (Rangel \& Velásquez, 1997). 


\section{RESULTADOS Y DISCUSIÓN}

Aspectos de composición florística. Se encontraron 523 individuos, distribuidos en 69 especies, representadas en 36 géneros y 25 familias, de las cuales, 24 son Angiospermas (22 dicotiledóneas y 2 monocotiledóneas) y 1 , Pteridophyta. Los índices de diversidad Alpha (a) y Beta ( $\beta$ ) empleados evidenciaron tres comunidades vegetales diferentes, acompañadas de una baja a muy baja dominancia de especies, lo que se representó en un alta a muy alta diversidad de táxones (Tabla 1) (Figura 2). La diversidad registrada podría ser explicada por medio de la teoría del disturbio intermedio, que sugiere que la máxima diversidad de una comunidad se alcanza con intensidades medias de disturbio (Sousa, 1984). En los primeros estados de sucesión, las comunidades son más fluctuantes, haciendo que su diversidad varíe, mientras que, en estados más conservados, los ecosistemas están en un mayor equilibrio, induciendo a que las especies y las familias se estabilicen (Villareal et al. 2004).
A nivel específico, las tres parcelas estudiadas presentaron diferentes abundancias para táxones mayores a $2,5 \mathrm{~cm}$ de DAP (Tabla 1). Las familias y los géneros con mayor número de especies en la parcela 1, de 0,1ha, correspondieron con: Melastomataceae (6), Clusiaceae (2), Asteraceae (2), Rubiaceae (2), Miconia (3), Axinaea (2) y Palicourea (2). Por su parte, la segunda parcela, de 0,1 ha, presentó a las familias Melastomataceae (5), Cyatheaceae (2), Cunoniaceae (2) y los géneros Axinaea (2), Cyathea (2), Miconia (2) y Weinmannia (2), con mayor representatividad de especies. Por último, la parcela 3, de 0,05ha, destacó a Melastomataceae (3) y Clethraceae (2), como las familias con presencia de más de una especie, siendo el género Clethra, el de mayor representatividad, con 2 especies.

La composición encontrada en este estudio corresponde con la flora nativa de la región Andina, por encima de los $2.600 \mathrm{~m}$ de altitud (Cuatrecasas, 1958; Cleef et al. 1983; Rangel et al. 1997; Preciado, 2000). Se hallaron semejanzas con los resultados florísticos de Avella

Tabla 1. Variables de diversidad analizadas en las tres parcelas de bosque Andino, localizadas en la vereda Potrero Grande, ChipaqueCundinamarca.

\begin{tabular}{|c|c|c|c|}
\hline Variable & $\mathbf{P 1 ( 2 9 0 0 )}$ & $\mathbf{P 2}(\mathbf{2 8 4 0 )}$ & $\mathbf{P 3 ~ ( 2 6 7 0 )}$ \\
\hline Especies & 28 & 30 & 11 \\
\hline Individuos & 118 & 254 & 151 \\
\hline Simpson 1-D & 0,946 & 0,918 & 0,856 \\
\hline Shannon H & 3,083 & 2,792 & 2,115 \\
\hline
\end{tabular}

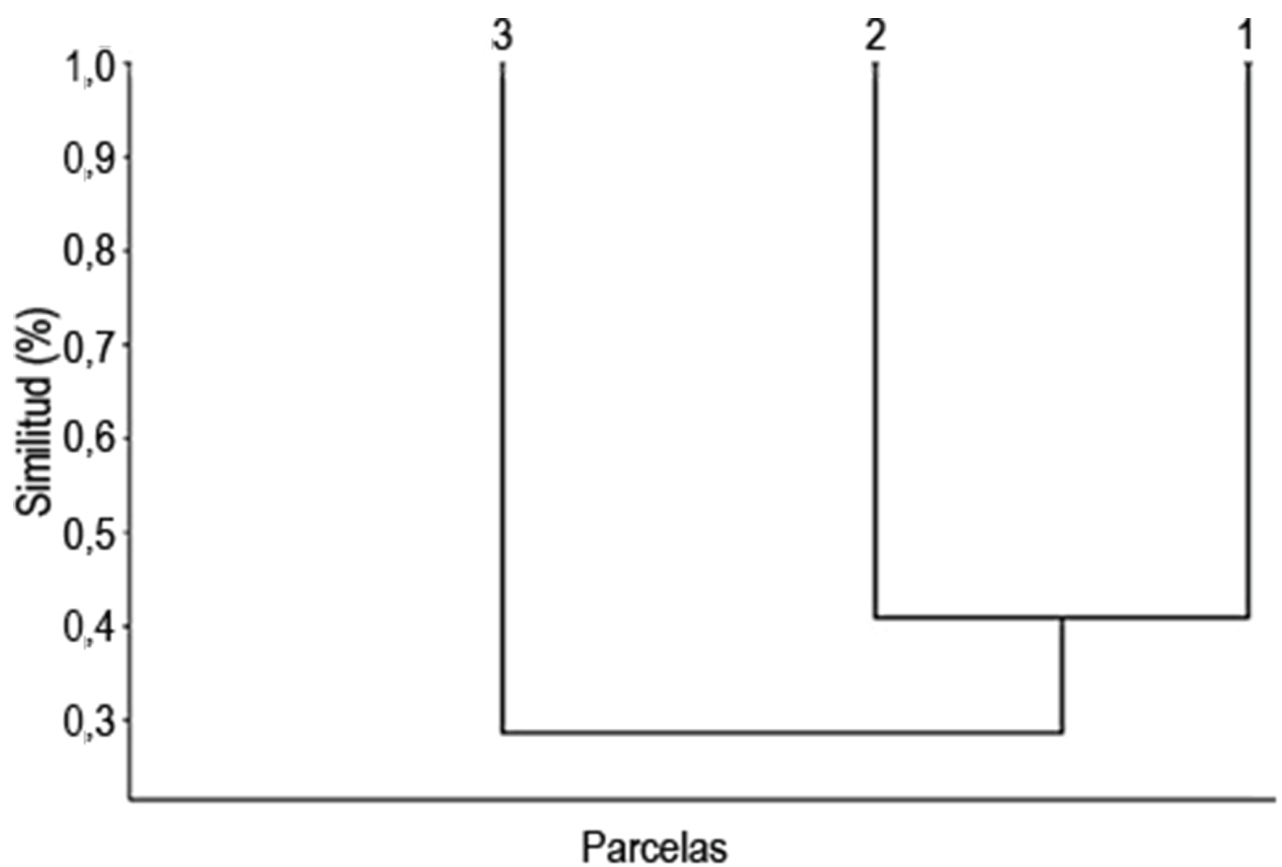

Figura 2. Relación de similaridad a partir del índice de Bray-Curtis para las tres parcelas de bosque Andino, localizadas en la vereda Potrero Grande, Chipaque-Cundinamarca. 
\& Ávila (2017), Phillips \& Miller (2002) y Álvarez et al. (2007). Cabe mencionar que, en comparación con las especies identificadas en el trabajo de Camelo et al. (2017), ciertas especies encontradas en el presente estudio corresponden a especies de tipo generalista, con alta capacidad de colonizar rápidamente distintos hábitats, por lo tanto, las comunidades con alta dominancia de las especies Cletbra fagifolia, Drimys granadensis, Tibouchina lepidota y Myrisne coriacea, pertenecen a fases más tempranas de la sucesión ecológica. Sumado a esto, los factores abióticos, como el régimen de lluvias, la irradiación solar, el microclima, las sequías estacionales, la altitud y el déficit de nutrientes a causa del uso intensivo del suelo que, para la zona de estudio radicó principalmente en actividades agropecuarias, generan alteraciones en la recuperación de la composición florística (Hooper, 2008).

Los ecosistemas andinos de Suramérica septentrional presentan similitudes en sus ensamblajes de composición de especies relativamente estables a lo largo del último periodo glacial, incluso, a pesar de cambios graduales a escala de paisaje, como se podría inferir a partir de los estudios de Cárdenas et al. (2014) y Loughlin et al. (2018), quienes encontraron, mediante estudios de paleopalinología en el flanco oeste de la cordillera de los Andes ecuatorianos, elementos comunes a la flora colombiana, entre los cuales, cabe destacar, por su abundancia a las familias Melastomataceae, Cunoniaceae, Asteraceae, Actinidiaceae, Adoxaceae, Aquifoliaceae, Clethraceae, Winteraceae y Chloranthaceae.

Particularmente, para las familias Clethraceae, Melastomataceae y Asteraceae, los estudios de paleopalinología realizados por Bush et al. (2015) y Valencia et al. (2016), en el Parque Nacional Manu, localizado en un bosque andino peruano, señalan que existe un indicador de recambio composicional, desde taxones propios de bosque maduro hacia elementos de estadios sucesionales más tempranos; esto indicaría, que el bosque presente en el área de estudio de Chipaque, se encuentra en una fase sucesional inicial a tardía. Asimismo, se registró que la riqueza florística del presente estudio tiene similitudes con los patrones de composición de los bosques húmedos montanos del Perú, en donde Ledo et al. (2012) reportan, como géneros dominantes en los estratos altos del dosel del bosque, a Clusia, Meliosma, Oreopanax y Weinmannia y, en los estratos más bajos del dosel, géneros como Myrcianthes, Palicourea y Solanum, todos presentes en los levantamientos realizados en Chipaque.

Aspectos estructurales. La distribución diamétrica del total de individuos hallados en las tres parcelas de vegetación de bosque montano presentó un comportamiento en " $\mathrm{j}$ invertida", lo cual, señala que, en mayor frecuencia, los individuos se agruparon en las categorías diamétricas más bajas, donde la clase más representativa correspondió a los 2,5 a 7,5cm de DAP, disminuyendo gradualmente hacia los mayores intervalos (Figura 3). Los mayores valores registrados por encima de $42,5 \mathrm{~cm}$ de DAP, se ubicaron en individuos de las especies Clusia multiflora (43,3cm), Centronia brachycera (45,5cm) y Prunus opaca (47,7cm). Al respecto, Lamprecht (1990) y Cantillo et al. (2004) señalan este comportamiento, como una tendencia normal para bosques naturales, con buen estado de desarrollo, lo que indica, un proceso sucesional activo, para el ecosistema estudiado.

A nivel de cada levantamiento, la variable altura presentó variaciones. Las parcelas 1 y 2 mostraron una mayor distribución de los individuos en los rangos encontrados para la variable, contrario a lo evidenciado en la parcela 3 , que obtuvo la mayor acumulación de individuos, en un único rango de 6,7 a 9,2. Entre los individuos más altos observados en los levantamientos, se registraron Prunus opaca, con 25m; Ageratina asclepiadea, con 24m; Clusia multiflora, con $19 \mathrm{~m}$ y Morella pubescens, con 19m. En su mayoría, estas especies presentaron los DAP más significativos (Figura 4).

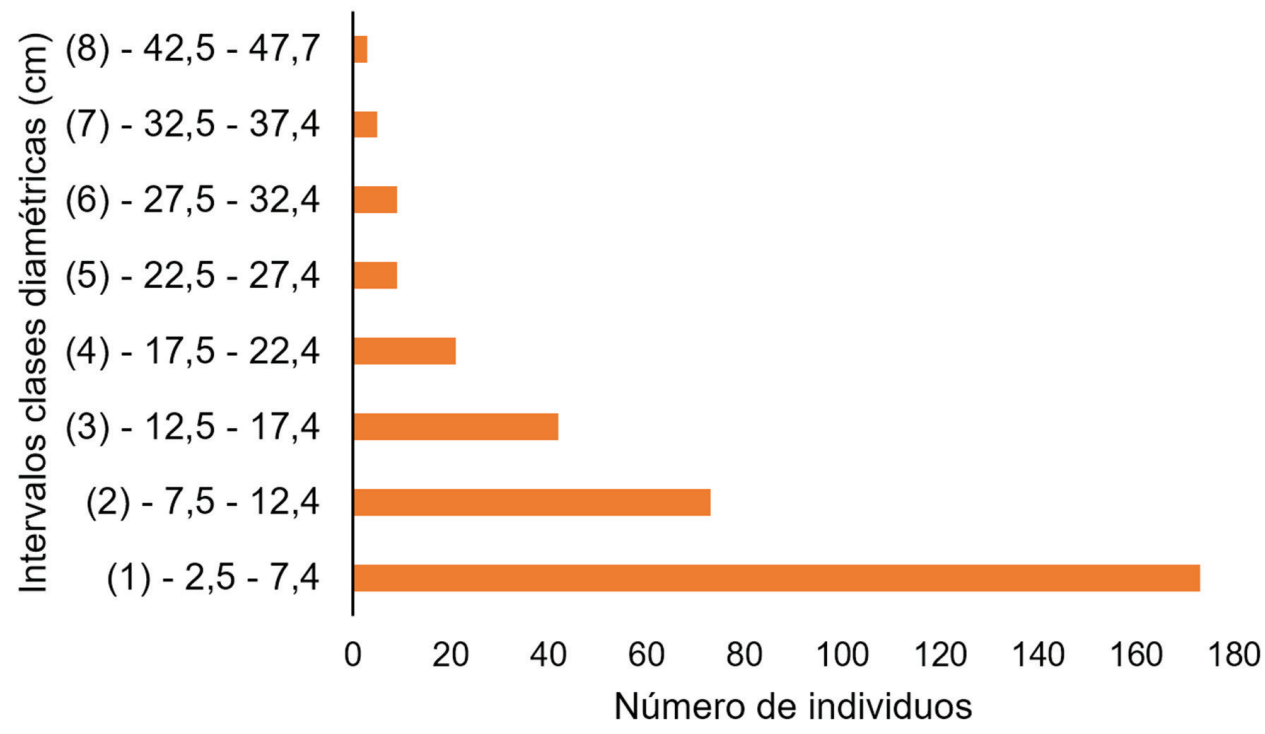

Figura 3. Distribución diamétrica de individuos con DAP $>2.5$ para las tres parcelas estudiadas de un bosque montano del municipio de Chipaque-Cundinamarca. 


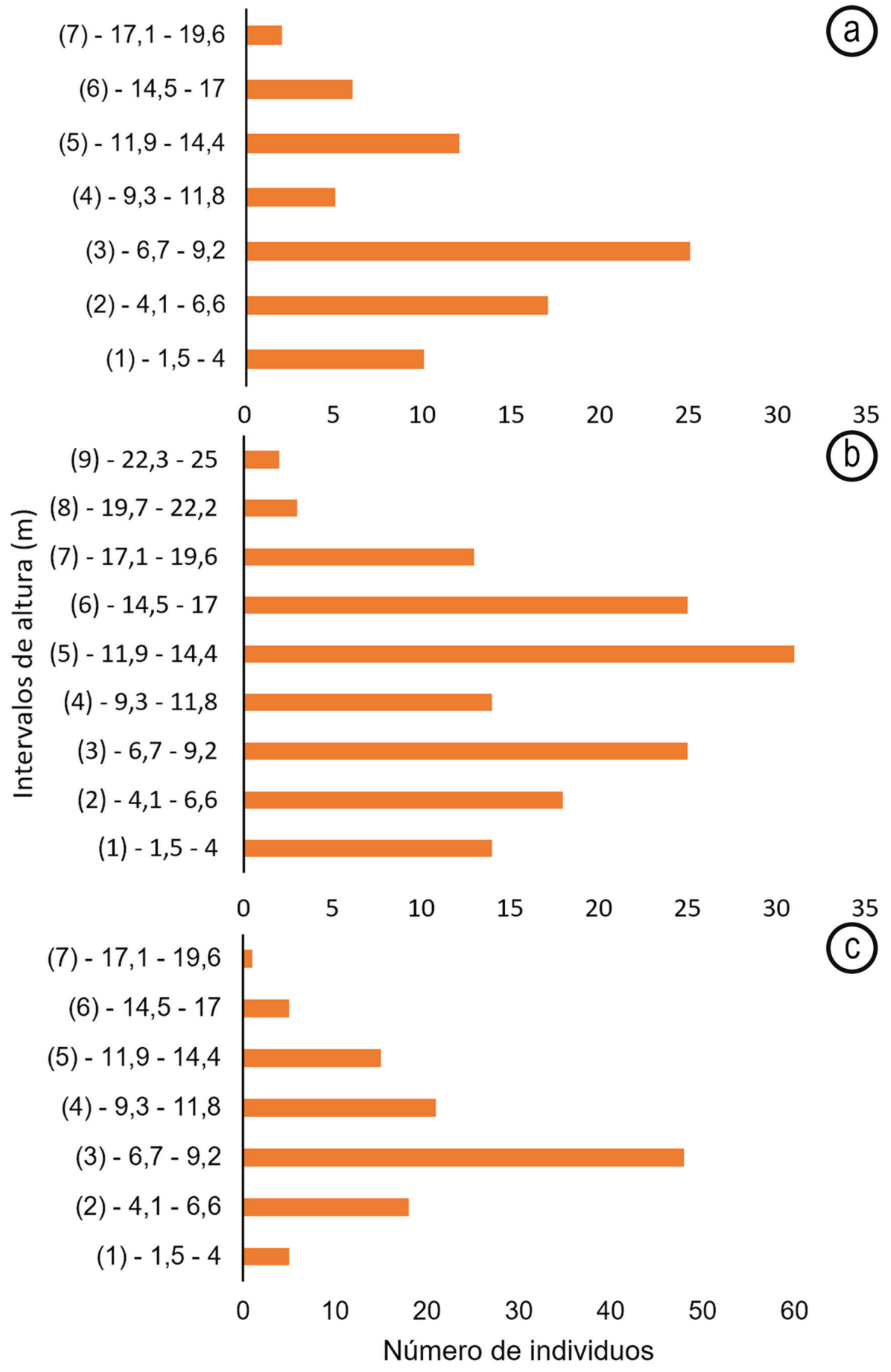

Figura 4. Distribución de frecuencias de intervalos de alturas para las tres parcelas estudiadas en un bosque Andino del municipio de Chipaque-Cundinamarca. a. Parcela 1; b. Parcela 2; c. parcela 3.

En los levantamientos de la parcela 1 y 3 fue posible apreciar ciertas similitudes en la dominancia de las especies, como Weinmannia tomentosa y Clusia multiflora, siendo, esta última, común en suelos drenados de alta humedad en asociación ecológica con especies del género Weinmannia, en donde presenta un papel subdominante en encenillales y es precursora de transiciones a otros tipos de bosques, pues es un inductor preclimático tardiseral (DAMA, 2000). Para el caso de Weinmannia tomentosa, Lequerica et al. (2017) señalan que esta especie posee alta durabilidad en el bosque y es de fácil propagación, por lo que podría ser considerada en procesos de restauración asistida. 
El Índice de Valor de Importancia para los tres levantamientos evidenció que las especies con mayor importancia ecológica fueron Clusia multiflora y Centronia brachycera, presentando la codominancia de diferentes especies, como Meliosma bogotana, Axinaea macrophylla y Tibouchina lepidota. En las parcelas 1 y 3 fue posible evidenciar que compartían otra especie codominante, correspondiente a Weinmannia tomentosa, contrario a lo que se observó en la parcela 2, en donde la especie Palicourea apicata obtuvo la tercera mayor representatividad (Tabla 2). Las especies reportadas con mayor IVI responden a un amplio rango altitudinal de distribución en los Andes tropicales, en regiones de vida andina y alto andina (DAMA, 2000; Lozano \& Alvear, 2001; Mendoza \& Fernández, 2012). Particularmente, la especie Meliosma bogotana, se presenta en bosques de las cordilleras, desde el nivel del mar hasta los límites altitudinales del bosque andino, en el territorio Nacional (Cuatrecasas \& Idrobo, 1955).
Los valores registrados para el IVI establecen pautas importantes en materia de selección de especies, en un contexto de restauración ecológica, pues permiten obtener información de la flora nativa, en respuesta a diferentes estadios de sucesión; esto, permitiría enriquecer acciones de restauración, como las plantaciones escalonadas, teniendo en cuenta las demandas de luz y de recursos de cada especie (Knowles \& Parrotta, 1995). Adicional a ello, este proceso activo de restauración facilitaría la reposición de los servicios del ecosistema en comprensión de las interacciones que tienen lugar entre los diversos usos de la tierra (Sabogal et al. 2015).

Este estudio permitió incrementar el conocimiento cuantitativo y cualitativo de los bosques Andinos asociados al municipio de Chipaque, en el departamento de Cundinamarca. La heterogeneidad florística encontrada en la vegetación secundaria analizada

Tabla 2. Especies dominantes y codominantes, de acuerdo con el IVI para los tres levantamientos en un bosque montano del municipio de Chipaque- Cundinamarca.

\begin{tabular}{|c|l|c|c|c|c|}
\hline Parcela & \multicolumn{1}{|c|}{ Especie } & DeR (\%) & DR (\%) & FR (\%) & IVI \\
\hline \multirow{4}{*}{1} & Clusia multiflora & 9,4117647 & 39,710082 & 11,538462 & 60,660308 \\
\cline { 2 - 6 } & Meliosma bogotana & 11,764706 & 21,576319 & 11,538462 & 44,879487 \\
\cline { 2 - 6 } & Weinmannia tomentosa & 7,0588235 & 10,611551 & 9,6153846 & 27,285759 \\
\hline \multirow{3}{*}{2} & Centronia brachycera & 20,547945 & 26,519252 & 12,068966 & 59,136163 \\
\cline { 2 - 6 } & Axinaea macrophylla & 14,383562 & 16,347871 & 17,241379 & 47,972812 \\
\cline { 2 - 6 } & Palicourea apicata & 23,972603 & 7,6921148 & 12,068966 & 43,733683 \\
\hline \multirow{3}{*}{3} & Clusia multiflora & 33,628319 & 19,157903 & 14,634146 & 67,420368 \\
\cline { 2 - 6 } & Tiboucbina lepidota & 14,159292 & 26,454387 & 19,512195 & 60,125874 \\
\cline { 2 - 6 } & Weinmannia tomentosa & 12,389381 & 16,305756 & 12,195122 & 40,890258 \\
\hline
\end{tabular}

proporciona conocimiento científico estratégico, para explorar diferentes alternativas de propagación e introducción de especies nativas, que resulten propicias para integrar en procesos de restauración ecológica, a nivel de paisaje.

Agradecimientos: Los autores del presente estudio expresan su agradecimiento a la subdirección Científica del Jardín Botánico, por facilitar los recursos físicos y el personal operativo. Extendemos nuestros agradecimientos al personal científico y administrativo del Herbario del JBB, en donde reposa el material vegetal colectado. De igual forma, agradecemos al técnico Enoc Sánchez, a Javier Mauricio Varón y a los expertos que fueron consultados, para la identificación del material vegetal: Dra. Mireya Patricia Córdoba y Gustavo Morales. Finalmente, agradecemos a Korina OcampoZuleta, por la revisión y comentarios efectuados en el Documento. Conflictos de intereses: El manuscrito fue elaborado y revisado con la participación de todos los autores, quienes declaramos que no existe ningún conflicto de intereses que ponga en riesgo la validez de los resultados presentados. Financiación: Este estudio contó con el apoyo financiero del proyecto 1121 Investigación para la conservación de los ecosistemas y la flora de Bogotá D.C., del Jardín Botánico de Bogotá José Celestino Mutis.

\section{REFERENCIAS}

1. ALCALDÍA MUNICIPAL DE CHIPAQUE CUNDINAMARCA. 2000. Esquema de Ordenamiento Territorial del Municipio de Chipaque. Colombia. Disponible desde Internet en: http://cdim.esap.edu.co/BancoConocimiento/C/chipaque_-_cundinamarca____eot_-_2000/chipaque_-_cundinamarca____eot_-_2000.asp [con acceso el $10 / 5 / 2018]$.

2. Álvarez, E.; COGOllo, A.; MELO, O.; ROJAS, E.; SÁNCHEZ, D.; VELÁSQUEZ, O.; JIMÉNEZ, E.; BENÍTEZ, D.; VELÁSQUEZ, C.; SERNA, M.; PÉREZ, J.; CARDONA, F; DEVIA, W. 2007. Monitoreo de los Andes colombianos (2000-3000 msnm) a través del 
establecimiento de parcelas permanentes. En: Armenteras, D.: Rodríguez, N. (eds.), Monitoreo de los ecosistemas andinos 1985 -2005: Síntesis y perspectivas. Instituto de Investigación Alexander von Humboldt (Bogotá). p.75-89.

3. ALVEAR, M.; BETANCUR, J.; FRANCO-ROSSELL, P. 2010. Diversidad florística y estructura de remanentes de bosque andino en la zona de amortiguación del parque nacional natural los nevados, cordillera central colombiana. Caldasia (Colombia). 32(1):39-63.

4. ARMENTERAS, D.; ESPELTA, J.M.; RODRÍGUEZ, N.; RETANA, J. 2017. Deforestation dynamics and drivers in different forest types in Latin America: Three decades of studies (1980-2010). Global Environmental Change. (Holland). (46):139-147. https://doi.org/10.1016/j. gloenvcha.2017.09.002

5. AVELLA, A.; ÁVILA, F. 2017. La flora vascular de los bosques de roble (Fagaceae) en Colombia: aproximación inicial. En: Rangel-Ch., J.O. (ed.), Colombia Diversidad Biótica XV. Los bosques de robles (Fagaceae) en Colombia. Composición florística, estructura, diversidad y conservación Universidad Nacional de Colombia - Instituto de Ciencias Naturales (Bogotá). p.311.

6. BUSH, M.B.; REYNOLDS, A.; URREGO, D.H.; VALENCIA, B.; CORREA-METRIO, A.; ZIMMERMANN, M.; SILMAN, M.R. 2015. Fire and climate: contrasting pressures on tropical Andean timberline species. J. Biogeography. (United Kingdom). 42(5):938-950. https:/ / doi.org/10.1111/jbi.12470

7. BUSSMANN, R.W. 2004. Regeneration and succession patterns in African, Andean and Pacific Tropical Mountain Forests: The role of natural and anthropogenic disturbance. Lyonia A J. Ecology and application. (U.S.A). 6(1):93-111.

8. CAmelo, O.; URrego, L.; OrRegO, S. 2017. Environmental and socioeconomic drivers of woody vegetation recovery in a human-modified landscape in the Rio Grande basin (Colombian Andes). Restoration Ecology. (United Kingdom). 25(6):912-921. https://doi. org/10.1111/rec. 12514

9. CANTILlO, E.; RODRÍGUEZ, R.; AVELLA, A. 2004. Diversidad y caracterización florística estructural de la vegetación arbórea en la Reserva Forestal Carpatos (Guasca, Cundinamarca). Colombia Forestal. (Colombia). 8(17):5-21. https://doi.org/10.14483/udistrital.jour. colomb.for.2004.1.a01

10. CÁRDENAS, M.; GOSLING, W.; PENNINGTON, R.; POOLE, I.; SHERLOCK, S.; MOTHES, P. 2014. Forests of the tropical eastern Andean flank during the middle Pleistocene. Palaeogeography, Palaeoclimatology,
Palaeoecology. (Holland). 393:76-89. https://doi. org/10.1016/j.palaeo.2013.10.009

11. CHAZDON, R.L. 2003. Tropical forest recovery: legacies of human impact and natural disturbances. Perspectives in Plant Ecology, Evolution and Systematics. (Holland). 6:5171. https://doi.org/10.1078/1433-8319-00042

12. CLEEF, A.M.; RANGEL-CH, J.O.; SALAMANCA, S. 1983. Reconocimiento de la vegetación de la parte alta del transecto Parque Nacional Natural Los Nevados. En: Van der Hammen, T.; Pérez, A.; Pinto, P. (eds.), La Cordillera Central de Colombia, transecto Parque Nacional Natural Los Nevados. Estudios de Ecosistemas Tropandinos, J. Cramer (Berlín). 1 p.150-173.

13. CUATRECASAS, J.; IDROBO, J. 1955. El Género Meliosma en Colombia. Caldasia. (Colombia). 7(33):187-211.

14. CUATRECASAS, J. 1958. Aspectos de la vegetación natural en Colombia. Revista de la Academia Colombiana de Ciencias Exactas, Físicas y Naturales. (Colombia). 10(40):221-268.

15. DEPARTAMENTO TÉCNICO ADMINISTRATIVO DEL MEDIO AMBIENTE - DAMA. 2000. Protocolo Distrital de Restauración. Guía para la restauración de ecosistemas nativos en las áreas rurales de Santa Fé de Bogotá. Departamento Técnico Administrativo de Medio Ambiente. Alcaldía Mayor de Santa Fé de Bogotá. Fundación Bachaqueros. (Bogotá). p.288.

16. FOOD AND AGRICULTURE ORGANIZATION FAO. 2015. Global Forest Resources Assessment, Desk Reference. Food and Agriculture Organization of the United Nations (Roma). 253p.

17. GUARIGUATA, M.R.; OSTERTAG, R. 2001. Neotropical secondary forest succession: changes in structural and functional characteristics. Forest Ecology and Management. (Holland). 148(1-3):185-206. https://doi.org/10.1016/ S0378-1127(00)00535-1

18. HAMMER, Ø.; HARPER, D.A.T.; RYAN, P.D. 2001. PAST: Paleontological Statistics Software Package for Education and Data Analysis. Palaeontologia Electronica. (U.S.A). 4(1):1-9.

19. HOOPER, E. 2008. Factors Affecting the Species Richness and Composition of Neotropical Secondary Succession: A Case Study of Abandoned Agricultural Land in Panama. En: Myster, R.W. (Ed.). Post-agricultural succession in the Neotropics. Springer (New York). p.141-163.

20. INSTITUTO DE HIDROLOGÍA, METEOROLOGÍA Y ESTUDIOS AMBIENTALES - IDEAM. 2018. Consulta y descarga de datos hidrometeorológicos. 
Disponible desde internet en: http://dhime.ideam.gov. co/atencionciudadano/ (con acceso el 12/06/2018).

21. KNOWLES, O.H.; PARROTTTA, J.A. 1995. Amazonian forest restoration: an innovative system for native species selection based on phonological data and field performance indices. Commonwealth Forestry Review. (United Kingdom). 74:230-243.

22. LAMPRECHT, H. 1990. Silvicultura en los trópicos. Los ecosistemas forestales en los bosques tropicales y sus especies arbóreas posibilidades y métodos para un aprovechamiento sostenido. (GTZ) GMBH, Eschborn, Alemania. 335p.

23. LEDO, A.; CONDÉS, S.; ALBERDI, I. 2012. Forest biodiversity assessment in Peruvian Andean Montane cloud forest. J. Mountain Science. (China). 9(3):372-384. https://doi. org/10.1007/s11629-009-2172-2

24. LEQUERICA, M.; BERNAL, M.; STEVENSON, P. 2017. Evidencia de direccionalidad del proceso de sucesión temprana del bosque altoandino. Colombia Forestal. (Colombia). 20(1):63-84. https://doi.org/10.14483/ udistrital.jour.colomb.for.2017.1.a06

25. LOUGHLIN, N.; GOSLING, W.; COE, A.; GULLIVER, P.; MOTHES, P.; MONTOYA, E. 2018. Landscape-scale drivers of glacial ecosystem change in the montane forests of the eastern Andean flank, Ecuador. Palaeogeography, Palaeoclimatology, Palaeoecology. (Holland). 489:198-208. https://doi.org/10.1016/j.palaeo.2017.10.011

26. LOZANO, G.; ALVEAR, M. 2001. Novedades en Axinaea y Meriania (Melastomataceae) de Colombia. Caldasia. (Colombia). 23(1):147-152.

27. MENDOZA, H.; FERNÁNDEZ, J.L. 2012. Novedades en Centronia y Meriania (Merianieae, Melastomataceae) y revisión taxonómica de Meriania grupo Brachycera. Anales del Jardín Botánico de Madrid. (España). 69(2):259-294.

28. MORENO, C. 2001. Métodos para medir la biodiversidad. M. \& T. Manuales y Tesis SEA. (Zaragoza). 1:84p.

29. MORIN, P.J. 2011. Comunity Ecology. Second Edition. Wiley -Blackwell (Oxford). 407p.

30. PHILLIPS, O.L.; MILLER, J.S. 2002. Global patterns of plant diversity: Alwyn H. Gentry's forest transect data set. Monographs in Systematics Botany from the Missouri Botanical Garden. (U.S.A). 89:1-317.

31. PICKETT, S.T.A.; WHITE, P.S. 1985. Natural disturbance and patch dynamics: An introduction. Pickett, S.T.A.; White
P.S. (eds). The Ecology of Natural Disturbance and Patch Dynamics. Academic Press (Orlando, Florida). p.3-4.

32. PRECIADO, A.P. 2000. La estructura ecológica principal de la Sabana de Bogotá. Boletín Geografía (Colombia). 44(131):1-37.

33. QUESADA, M.; SÁNCHEZ ASOFEIFA, G.A.; ALVAREZANORVE, M.; STONER, K.E.; AVILA-CABADILLA, L.; CALVO-ALVARADO, J.; GAMON, J. 2009. Succession and management of tropical dry forests in the Americas: Review and new perspectives. Forest Ecology and Management. (Holland). 258(6):1014-1024. https://doi. org/10.1016/j.foreco.2009.06.023

34. RANGEL, J.O.; VELÁZQUEZ, A. 1997. Métodos de estudio de la vegetación. En: Rangel-Ch., J.O.; Lowry, P.; M. Aguilar, M. (eds). Colombia Diversidad Biótica II. Tipos de vegetación en Colombia. Universidad Nacional de Colombia (Bogotá). p.59-87.

35. RANGEL-CH, J.O.; LOWY, P.; AGUILAR, M. 1997. Distribución de los tipos de vegetación en las regiones naturales de Colombia. Aproximación inicial. En: RangelCh., J.O.; Lowry-C, P.; Aguilar-P., M. (eds). Diversidad Biótica II. Tipos de Vegetación en Colombia. Universidad Nacional de Colombia-Instituto de Ciencias Naturales, Instituto de hidrología, Meteorología y estudios Ambientales (IDEAM)-Ministerio del Medio Ambiente, Comité de Investigaciones y Desarrollo Científico-CINDEC. U.N, Academia Colombiana de Ciencias Exactas, Físicas y Naturales (Bogotá). p.403-436.

36. SABOGAL, C.; BESACIER, C.; MCGUIRE, D. 2015. Restauración de bosques y paisajes: conceptos, enfoques y desafíos que plantea su ejecución. UNASYLVA 245. Rev. Internal sobre bosques y actividades e industrias forestales (Italia). 66:3-10.

37. SÁENZ, G.P.; FINEGAN, B. 2000. Monitoreo de la regeneración natural con fines de manejo forestal. Manejo Forestal Tropical (CATIE) (Turrialba.). 15:8p.

38. SOUSA, W.P. 1984. The role of disturbance in natural communities. Annual Review of Ecology and Systematics. 15:353-391.

39. VALENCIA, B.; MATTHEWS-BIRD, F.; URREGO, D.; WILLIAMS, J.; GOSLING, W.; BUSH, M. 2016. Andean microrefugia: testing the Holocene to predict the Anthropocene. New Phytologist (United Kingdom). 212(2):510-522. https://doi.org/10.1111/nph.14042

40. VILlAREAL, H.M.; ÁlVAREZ, M.; CÓRDOBACÓRDOBA, S.; ESCOBAR, F; FRAGUA, G.; GAST, F; 
MENDOZA-CIFUENTES, H.; OSPINA, M.; UMAÑA, A.M. 2004. Manual de métodos para el desarrollo de inventarios de biodiversidad. Programa de inventarios para la biodiversidad. Instituto de Investigación de Recursos Biológicos Alexander von Humboldt. (Bogotá). 236p. Disponible desde Internet en: http://hdl.handle. net/20.500.11761/31419(con acceso el 10/5/2018)
41. WHITMORE, T.C. 1998. A Pantropical perspective on the ecology that underpins management of tropical secondary rain forests. En: Guariguata, M.R.; Finegan, B. (eds.). Ecology and management of tropical secondary forest: science, people and policy. CATIE (Costa Rica). p.19-34. 